\title{
Glycochenodeoxycholic Acid Inhibits Calcium Phosphate Precipitation In Vitro by Preventing the Transformation of Amorphous Calcium Phosphate to Calcium Hydroxyapatite
}

Sui-Min Qiu, Gary Wen, Nobuyuki Hirakawa, Roger D. Soloway, Nan-Kang Hong, and Roger S. Crowther

Division of Gastroenterology, Department of Internal Medicine, University of Texas Medical Branch, Galveston, Texas 77550

\begin{abstract}
Calcium hydroxyapatite can be a significant component of black pigment gallstones. Diverse molecules that bind calcium phosphate inhibit hydroxyapatite precipitation. Because glycine-conjugated bile acids, but not their taurine counterparts, bind calcium phosphate, we studied whether glycochenodeoxycholic acid inhibits calcium hydroxyapatite formation. Glycochenodeoxycholic acid $(2 \mathrm{mM})$ totally inhibited transformation of amorphous calcium phosphate microprecipitates to macroscopic crystalline calcium hydroxyapatite. This inhibition was not mediated by decreased $\mathrm{Ca}^{2+}$ activity. Taurocholic acid (2$12 \mathrm{mM}$ ) did not affect hydroxyapatite formation, but antagonized glycochenodeoxycholic acid. Both amorphous and crystalline precipitates contained a surface fraction relatively rich in phosphate. The surface phosphate content was diminished by increasing glycochenodeoxycholic acid concentrations, and this relationship was interpreted as competition between bile acid and $\mathrm{HPO}_{4}^{2-}$ for binding sites on the calcium phosphate surface. A phosphate-rich crystal surface was associated with rapid transition from amorphous to crystalline states. These results indicate that glycochenodeoxycholic acid prevents transformation of amorphous calcium phosphate to crystalline hydroxyapatite by competitively inhibiting the accumulation of phosphate on the crystal embryo surface. (J. Clin. Invest. 1991. 88:1265-1271.) Key words: bile salts • biomineralization - calcification - Fourier transform infrared spectroscopy • gallstones
\end{abstract}

\section{Introduction}

Calcium salts of bilirubin, fatty acids, carbonate, and phosphate are important constituents of all classes of gallstones, although the predominant salt varies with the type of stone (1, 2). The fundamental drive for these salts to precipitate can be understood on a physicochemical basis as the solubility product for a particular salt being exceeded. However, many of

Part of this work has appeared in abstract form (1990. Gastroenterology. 98:A258).

Dr. Qiu is a Visiting Scientist from the Department of Hepatobiliary Surgery, First Affiliated Hospital, Xian Medical University, People's Republic of China.

Address reprint requests to Dr. Crowther, Gastroenterology Division, 4.106 McCullough Building, G-64, University of Texas Medical Branch, Galveston, TX 77550.

Received for publication 11 December 1990 and in revised form 24 June 1991.

J. Clin. Invest.

(c) The American Society for Clinical Investigation, Inc.

$0021-9738 / 91 / 10 / 1265 / 07 \quad \$ 2.00$

Volume 88, October 1991, 1265-1271 these species exist in normal bile in supersaturated concentrations, but do not precipitate. It is becoming apparent that other components of bile can interact with these species in ways that modify their precipitation, and specific modifying factors such as acidic glycoproteins (3) and bile acids (4) have been identified.

Our interest in the pathogenesis of pigment stones has led us to study factors capable of influencing the precipitation of calcium hydroxyapatite, $\mathrm{Ca}_{10}(\mathrm{OH})_{2}\left(\mathrm{PO}_{4}\right)_{6}$, (HAP), ${ }^{1}$ which, although it is a relatively uncommon gallstone component, can comprise up to $30 \%$ of the total mass of black pigment stones (2). Sutor and Percival (5) demonstrated that human common duct and gallbladder biles from subjects with cholesterol stones contained factors that inhibited calcium phosphate precipitation, and they proposed that bile salt/phospholipid micelles were responsible for this effect. Hydroxyapatite is formed from amorphous calcium phosphate (ACP) by dissolution and reprecipitation (6). ACP may have the stoichiometry of dicalcium phosphate dihydrate, $\mathrm{CaHPO}_{4} \cdot 2 \mathrm{H}_{2} \mathrm{O}$, or tricalcium phosphate, $\mathrm{Ca}_{3}\left(\mathrm{PO}_{4}\right)_{2} \cdot \mathrm{XH}_{2} \mathrm{O}$, depending on the formation conditions. It has been shown that bile acids can bind to ACP and that different bile acids have markedly different affinities (7). Glycine-conjugated dihydroxy bile acids showed the highest affinity, whereas all taurine conjugates showed almost no ability to bind.

We hypothesized that the effects seen by Sutor and Percival (5) could be caused by bile acids binding to calcium phosphate microprecipitates, thereby preventing further precipitation. To test this, we studied the effects of a glycine-conjugated dihydroxy bile acid, glycochenodeoxycholic (GCDC), and a taurine-conjugated trihydroxy bile acid, taurocholic (TC), which we predicted to be active and inactive, respectively, with respect to the inhibition of hydroxyapatite precipitation.

\section{Methods}

Materials. Calcium chloride, dibasic sodium phosphate, Trizma- $\mathrm{HCl}$, Trizma-base, calcium hydroxyapatite (type I), bile acids (sodium salts), and murexide were purchased from Sigma Chemical Co., St. Louis, MO. Diphenylhexatriene and tetrahydrofuran were obtained from Aldrich Chemical Co., Milwaukee, WI.

Stock solutions. Five stock solutions were prepared for use in all experiments: (a) $500 \mathrm{mM}$ Tris-HCl and Tris-base buffer, $\mathrm{pH} 7.50$; (b) $100 \mathrm{mM}$ calcium chloride; (c) $100 \mathrm{mM}$ dibasic sodium phosphate; $(d)$ $100 \mathrm{mM}$ bile acid; and (e) $1.5 \mathrm{M}$ sodium chloride.

Calcium phosphate formation. Solutions containing calcium or phosphate were prepared separately. Final concentrations in each solution were: $8 \mathrm{mM}$ calcium or $8 \mathrm{mM}$ phosphate, $50 \mathrm{mM}$ Tris buffer, $0-80$

1. Abbreviations used in this paper: ACP, amorphous calcium phosphate; CMC, critical micelle concentration; FTIR, Fourier transform infrared; GCDC, glycochenodeoxycholic acid; HAP, calcium hydroxyapatite; $I_{t}$, induction time; TC, taurocholic acid. 
$\mathrm{mM}$ bile acid, and sufficient sodium chloride to let $\mu=0.15$, in a total volume of $10 \mathrm{ml}$. Each solution was titrated to $\mathrm{pH} 7.50$, passed through a $0.22-\mu \mathrm{m}$ filter (Micro Separation Inc., Westborough, MA), and equilibrated at $37^{\circ} \mathrm{C}$ for $20 \mathrm{~min}$. During this time the $\mathrm{pH}$ fell from 7.50 to $7.31 \pm 0.02$. To start the reaction, we mixed equal volumes of calciumand phosphate-containing solutions, so that the initial concentration of each reactant was $4 \mathrm{mM}$. These concentrations were used by Sutor and Percival (5) and result in precipitation within a convenient time scale. The mixed solutions were placed in quartz cuvettes maintained at $37^{\circ} \mathrm{C}$, and the formation of calcium phosphate was monitored turbidimetrically at $220 \mathrm{~nm}(8)$ (model 4053 spectrophotometer, Biochrom, Cambridge, England). In some experiments an aliquot of $500 \mu \mathrm{l}$ was withdrawn after $22 \mathrm{~h}$ of incubation and centrifuged for $5 \mathrm{~min}$ at 11,000 $g$, and the supernatant total calcium and phosphate content was determined.

Determination of total calcium and phosphate. Total calcium was measured by atomic absorption spectrophotometry (model 5100, Perkin-Elmer Corp., Norwalk, CT) after dilution of samples with 5\% trichloracetic acid/ $1 \%$ lanthanum chloride. Phosphate was determined by conductivity measurement after ion exchange HPLC (Ionpac AS4A column, Dionex, Sunnyvale, CA) or by the molybdenum blue method (9).

Fourier transform infrared spectroscopy (FTIR). Calcium- and phosphate-containing solutions, with or without bile salts, were mixed and placed in an attenuated total reflectance cell fitted with a zinc selenide crystal (CIRcle cell, Spectra-Tech, Inc., Stamford, CT) and mounted in an FTIR spectrometer (model FTS 60, Digilab, Boston, MA). Spectra were collected at intervals for up to $72 \mathrm{~h}$. The spectrum of a reference solution, which consisted of $50 \mathrm{mM}$ Tris, $4 \mathrm{mM} \mathrm{Ca}^{2+}$, and $100 \mathrm{mM} \mathrm{NaCl}$, was manually subtracted from each sample spectrum to disclose the signal from the precipitate. Spectra were not corrected for phosphate because the absorbance from precipitates was at least 20 times greater than the absorbance of $4 \mathrm{mM}$ phosphate.

Determination of bile acid critical micelle concentration (CMC). The CMCs of bile acids were measured fluorimetrically (10). $1 \mu$ of 10 $\mathrm{mM}$ diphenylhexatriene in tetrahydrofuran was added to different concentrations of bile acids dissolved in a total volume of $2 \mathrm{ml}$ of Tris-buffered solution, $\mathrm{pH} 7.5, \mu=0.15$. Tubes were incubated in the dark at room temperature for $30 \mathrm{~min}$. Fluorescence was measured at an excitation wavelength of $358 \mathrm{~nm}$ and an emission wavelength of $430 \mathrm{~nm}$ (model LS-5B luminescence spectrometer, Perkin-Elmer Corp.).

Effect of bile acids on $\mathrm{Ca}^{2+}$ activity. Calcium activity was determined with the calcium-sensitive indicator murexide. The absorbance of calcium-containing solutions at 540 and $470 \mathrm{~nm}$ was measured against reference solutions that were of the same composition, including bile acid when appropriate, but were deprived of calcium (11). In this way the effect of any shift in the dye absorbance spectrum caused by bile acid-dye interaction was minimized. The difference in absorbance at the two wavelengths was linearly related to the logarithm of calcium activity.

\section{Results}

Calcium phosphate formation in the absence of bile salt. When the formation of calcium phosphate was monitored by turbidity measurement, a typical curve (Fig. 1) comprised three phases: (1) an initial steady increase in turbidity, which gradually slowed; (2) a sudden rapid increase in turbidity, which was initiated at $56.6 \pm 4.7 \mathrm{~min}$ (mean $\pm \mathrm{SD}, n=5$ ); and (3) a gradual decline in turbidity as large particles sedimented. When $\mathrm{pH}$ was monitored, there was an initial rapid fall of $\sim 0.03 \mathrm{pH}$ units that was probably caused by the formation of soluble $\mathrm{Ca}^{2+} / \mathrm{HPO}_{4}^{2-}$ complexes. Throughout the remainder of phase 1 the $\mathrm{pH}$ fell a further $0.01 \mathrm{pH}$ units, and then, simultaneously with the onset of phase 2 , the $\mathrm{pH}$ of the solution declined sharply (Fig. 1), indicating that the events of phase 2 involved

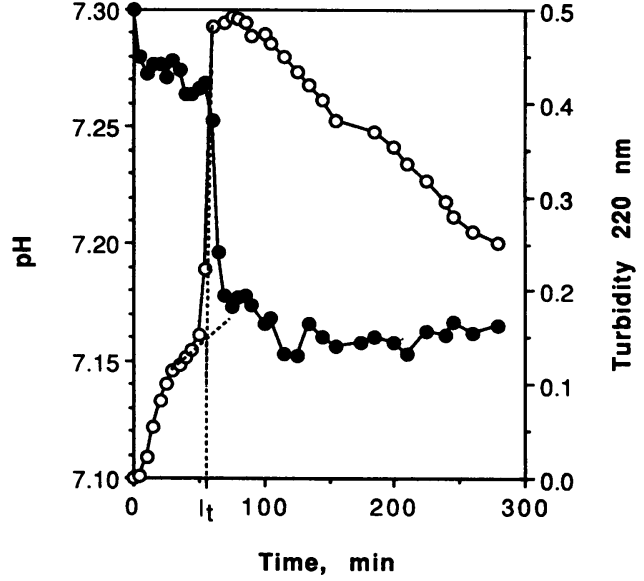

Figure 1. Formation of calcium phosphate monitored by turbidity and $\mathrm{pH} . \mathrm{Ca}^{2+}$ and phosphate ( $4 \mathrm{mM}$ final concentration of each) were mixed together at $\mathrm{pH} 7.3, \mu=0.15,37^{\circ} \mathrm{C}$, and formation of precipitate was monitored either by turbidity $(0)$ or $\mathrm{pH}(\bullet)$ measurements.

precipitation of additional calcium phosphate, not simply aggregation of existing material. These curves are similar to those seen by other investigators (12). The onset of rapid precipitation has been associated with the transformation of ACP to HAP and the time required for this to occur has been termed the induction time $\left(I_{t}\right)(12)$.

Effect of bile acids on the precipitation of calcium phosphate. GCDC at a concentration of $0.5 \mathrm{mM}$ reduced $I_{\mathrm{t}}$ by $\sim 15$ min, but with increasing GCDC concentrations $I_{\mathrm{t}}$ was progressively lengthened, until at a concentration of $2 \mathrm{mM}$ and above, phase 1 was prolonged for $5 \mathrm{~h}$, at which point the experiment was terminated (Fig. 2). Additional experiments showed that when the concentration of GCDC was $\geq 2 \mathrm{mM}$, there was no marked increase in turbidity and no visible precipitate, even after $4 \mathrm{~d}$ (not shown). Measurement of supernatant calcium

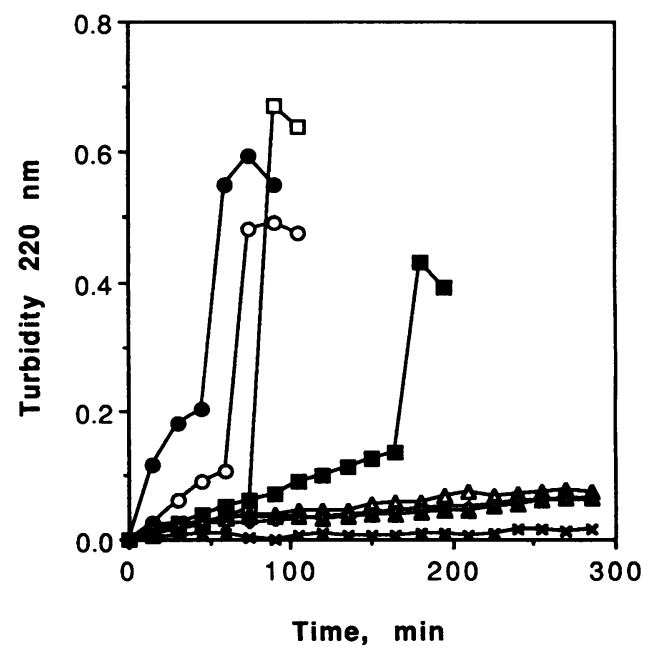

Figure 2. Effect of GCDC on calcium phosphate formation. $\mathrm{Ca}^{2+}$ and phosphate were mixed together under the standard conditions, either without (o) or with $0.5 \mathrm{mM}(\bullet), 1.0 \mathrm{mM}(\square), 1.5 \mathrm{mM}(\bullet), 2.0 \mathrm{mM}(\Delta)$, $4.0 \mathrm{mM}(\triangle), 8.0 \mathrm{mM}(\diamond)$, or $12.0 \mathrm{mM}(\times)$ GCDC; formation of precipitate was monitored by turbidity. The portions of the curves representing phase 3 have been omitted for clarity. 
and phosphate concentrations after $22 \mathrm{~h}$ demonstrated that, as well as increasing $I_{t}$, GCDC also decreased the total amount of calcium phosphate precipitated. In the absence of GCDC $7.52 \pm 0.03 \mu \mathrm{mol}$ of calcium was precipitated, and this was progressively reduced by $0.5 \mathrm{mM}(7.48 \pm 0.08), 1.0 \mathrm{mM}$ $(6.76 \pm 0.11), 1.5 \mathrm{mM}(6.29 \pm 0.03)$, and $2 \mathrm{mM}(0.74 \pm 0.60)$ GCDC. Similar results were obtained for phosphate precipitation. These experiments clearly show that GCDC causes concentration-dependent inhibition of calcium phosphate precipitation.

The effects of TC were minor compared with those of GCDC. There was some variation in $I_{t}$, but values were always within $20 \%$ of the control: $0 \mathrm{mM}, 55 \mathrm{~min} ; 2 \mathrm{mM}, 55 \mathrm{~min} ; 4$ $\mathrm{mM}, 45 \mathrm{~min}$; $9 \mathrm{mM}, 47 \mathrm{~min} ; 12 \mathrm{mM}, 62 \mathrm{~min}$.

The effect of GCDC and TC in combination was also studied. When a low concentration of GCDC $(1.5 \mathrm{mM})$ was mixed with increasing concentrations of TC $(2-12 \mathrm{mM}), I_{t}$ decreased exponentially as the amount of TC increased (Fig. 3). Interestingly, compared with $1.5 \mathrm{mM} \mathrm{GCDC}$ alone, the lowest TC concentration increased $I_{\mathrm{t}}$ (164 and $317 \mathrm{~min}$, respectively). Similar effects were observed with a higher GCDC concentration ( $4 \mathrm{mM}$ ) and TC varying between 2 and $12 \mathrm{mM}$ (Table I). Although TC concentrations of 2 and $4 \mathrm{mM}$ plus $4 \mathrm{mM} \mathrm{GCDC}$ postponed phase 2 longer than the duration of the experiment (500 $\mathrm{min}$ ), the turbidity readings during phase 1 indicated a faster precipitation rate than when $4 \mathrm{mM}$ GCDC alone was present (Table I).

CMC of bile acids. The CMCs of GCDC and TC were determined to be 2 and $8 \mathrm{mM}$, respectively. These values are consistent with previous reports (13) and were unaffected by the presence of either $4 \mathrm{mM} \mathrm{Ca}^{2+}$ or $4 \mathrm{mM}$ phosphate. However, since this method is relatively insensitive to submicellar aggregates, it cannot be ruled out that these ions may influence the size or number of submicellar oligomers. Experiments were also performed to study the state of aggregation of systems containing both GCDC and TC. When increasing amounts of TC were added to $1.5 \mathrm{mM}$ GCDC, TC became incorporated into mixed species aggregates, and the "CMC" for this mixed system was estimated to be $\sim 4 \mathrm{mM}$ total bile salts, but this value will depend upon the GCDC/TC ratio. Thus, the addition of $2 \mathrm{mM}$ TC to $1.5 \mathrm{mM} \mathrm{GCDC}$ increased the amount of system aggregation (either number or size of aggregates) and thereby provided more effective inhibition of HAP formation, resulting in the prolonged $I_{t}$ shown in Fig. 3. As more TC was added, the aggregates contained ever greater proportions of TC,

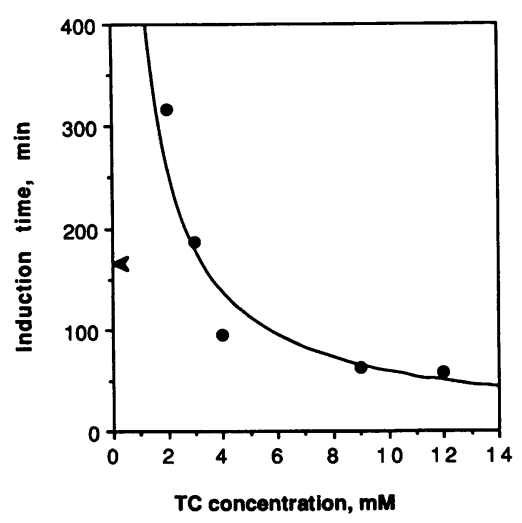

Figure 3. Correlation of induction time with GCDC/TC ratio. $\mathrm{Ca}^{2+}$ and phosphate were mixed together under the standard conditions together with $1.5 \mathrm{mM}$ GCDC and various concentrations of TC (2-12 mM). Values of $I_{t}$ were measured from plots of turbidity against time. Arrow indicates $I_{\mathrm{t}}$ for a system containing $1.5 \mathrm{mM} \mathrm{GCDC}$ and no TC.
Table I. Effect of GCDC and TC in Combination on Calcium Phosphate Formation

\begin{tabular}{ccc}
\hline$[\mathrm{GCDC}+\mathrm{TC}]$ & $\begin{array}{c}\text { Phase } 1 \\
\text { precipitation rate }\end{array}$ & \multicolumn{1}{c}{$\boldsymbol{I}_{\mathbf{t}}$} \\
\hline$m M$ & $\Delta O D / h$ & $\min$ \\
$0+0$ & 0.749 & 50 \\
$4+0$ & 0.022 & $>500$ \\
$4+2$ & 0.025 & $>500$ \\
$4+4$ & 0.044 & $>500$ \\
$4+9$ & 0.055 & 250 \\
$4+12$ & 0.076 & 82 \\
$0+12$ & 0.872 & 62 \\
& &
\end{tabular}

$\mathrm{Ca}^{2+}$ and phosphate (4 mM final concentration of each) were mixed together, with or without $4 \mathrm{mM} \mathrm{GCDC}$ and increasing concentrations of TC, at $\mathrm{pH} 7.3, \mu=0.15,37^{\circ} \mathrm{C}$; formation of precipitate was monitored by turbidity at $220 \mathrm{~nm}$. Precipitation rate and $I_{\mathrm{t}}$ were determined from plots of turbidity against time.

and this was responsible for the decreased inhibition seen in Fig. 3. This mechanism also operated when the GCDC concentration was $4 \mathrm{mM}$ and resulted in the attenuated inhibition shown in Table I.

Because potent inhibition was achieved at GCDC concentrations that approximated the $\mathrm{CMC}$, it seemed likely that small aggregates, perhaps dimers, rather than micelles, were responsible for this action. To test this possibility, we examined the effect of dehydrocholic acid on HAP formation, since dehydrocholic acid does not form micelles, but can form dimers (14). Under conditions of constant ionic strength, dehydrocholic acid caused modest inhibition of HAP formation: in the absence of dehydrocholic acid $4.72 \pm 0.08 \mu \mathrm{mol}$ of phosphate were precipitated at $24 \mathrm{~h}$, and this was progressively reduced by $20 \mathrm{mM}$ (4.61 \pm 0.09$), 40 \mathrm{mM}(4.21 \pm 0.01), 60 \mathrm{mM}$ (3.81 \pm 0.06$)$,

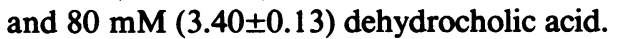

Effect of bile acids on $\mathrm{Ca}^{2+}$ activity. Under conditions of constant ionic strength, both TC and GCDC reduced $\mathrm{Ca}^{2+}$ activity, but GCDC was more effective than was TC (not shown). GCDC concentrations $<4 \mathrm{mM}$ had no measurable effect on $\mathrm{Ca}^{2+}$ activity, and GCDC concentrations of 4 and $20 \mathrm{mM}$ reduced activity by only $5 \%$ and $25 \%$, respectively. Even with a $25 \%$ reduction in activity, the solutions would remain highly supersaturated with respect to HAP, and the effect of GCDC must be mediated by an alternative mechanism. TC had a lesser effect on $\mathrm{Ca}^{2+}$ activity, and a $20 \mathrm{mM}$ solution decreased activity by only $6 \%$. Dehydrocholic acid reduced $\mathrm{Ca}^{2+}$ activity by $4 \%(20 \mathrm{mM}), 5 \%(40 \mathrm{mM}), 14 \%(60 \mathrm{mM})$, and $20 \%(80$ $\mathrm{mM})$. There was a close correlation between $\mathrm{Ca}^{2+}$ activity in the presence of dehydrocholic acid and the amount of HAP precipitated $\left(r^{2}=0.958, P<0.005\right)$.

FTIR studies of calcium phosphate formation. To demonstrate that phase 2 was associated with the formation of HAP, we analyzed the products of the reaction in situ by FTIR. In the absence of bile acid, the precipitation of calcium phosphate caused the intensity of the orthophosphate (P-O) absorption band at $1,150-950 \mathrm{~cm}^{-1}$ to increase with a time dependency that was very similar to the turbidity curves produced in earlier experiments, except that $I_{\mathrm{t}}$ was increased from 56 to $80 \mathrm{~min}$ (Fig. 4). The temperature of solutions in the CIRcle cell was 


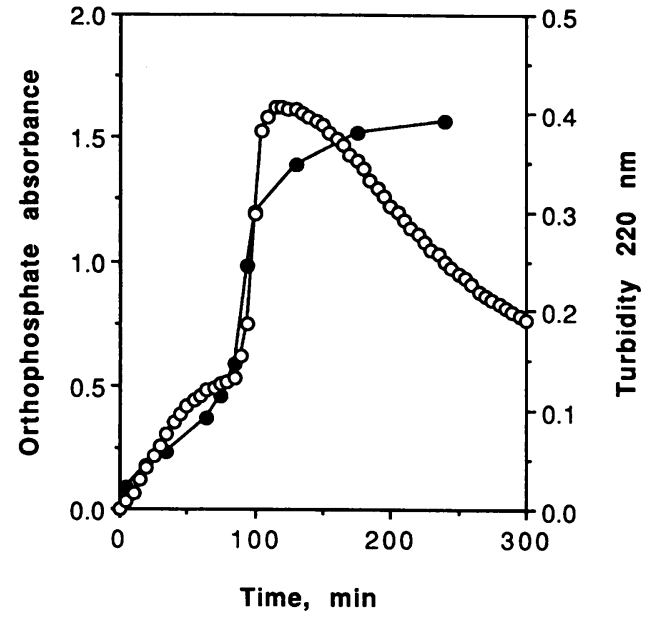

Figure 4. Formation of calcium phosphate monitored by FTIR spectroscopy. $\mathrm{Ca}^{2+}$ and phosphate were mixed together under the standard conditions, except that the temperature was $30^{\circ} \mathrm{C}$, and precipitation was monitored by either FTIR spectroscopy $(\bullet)$ or turbidity (o).

$30 \pm 1^{\circ} \mathrm{C}$, and when a turbidity experiment was repeated at $30^{\circ} \mathrm{C}$, the two sets of measurements correlated exactly (Fig. 4). Concurrently with the onset of phase 2 , the IR absorption spectrum of the calcium phosphate underwent a prominent change (Fig. $5 \mathrm{~A}$ ). Before phase 2 the orthophosphate absorption band was relatively symmetrical, indicating little distortion of the phosphate ions from an average tetrahedral symmetry, which is typical of ACP (15). But between 85 and $95 \mathrm{~min}$ (i.e., $\sim 5 \mathrm{~min}$ after $I_{t}$ ), the phosphate peak began to transform into a triplet (Fig. $5 A$ ), which indicated that certain $\mathrm{P}-\mathrm{O}$ vibrations were being selectively restricted by a newly forming crystal structure. By 240 min the spectrum was consistent with the bulk of the precipitate being HAP, as judged by comparison with an authentic HAP standard (Fig. $5 \mathrm{~A}$, inset). Thus phase 2 is associated with the emergence of HAP as the predominant form of precipitated calcium phosphate. The predominance of HAP could explain the greatly increased rate of precipitation at that time, because HAP is much less soluble than are other forms of calcium phosphate (16).

As would be predicted from earlier experiments, $12 \mathrm{mM}$ TC had little effect on the FTIR spectra, although $I_{t}$ and the appearance of the phosphate triplet were prolonged to $\sim 110$ min (not shown), which is consistent with the minor delaying effect previously seen with this concentration of TC (Table I). GCDC, however, had considerable concentration-dependent effects on the IR spectra as calcium phosphate precipitated. At $2 \mathrm{mM}$, GCDC completely prevented the appearance of the crystalline phosphate triplet, although amorphous material continued to form slowly (Fig. $5 \mathrm{~B}$ ). In contrast with the control, where the location of the maximum absorbance moved progressively to lower wavenumbers throughout the experiment, in the presence of $2 \mathrm{mM} \mathrm{GCDC}$ the peak did not shift at all, which suggests very effective inhibition of transformation. No crystalline material was detected at $3 \mathrm{~d}$, at which point the experiment was terminated. Thus, the effect of GCDC is mediated by preventing the transition of ACP microprecipitates to macroscopic crystalline HAP. With $1 \mathrm{mM} \mathrm{GCDC}$, the phosphate triplet appeared at $85 \mathrm{~min}$ (not shown), and $0.5 \mathrm{mM}$ GCDC accelerated the appearance of the triplet, which became

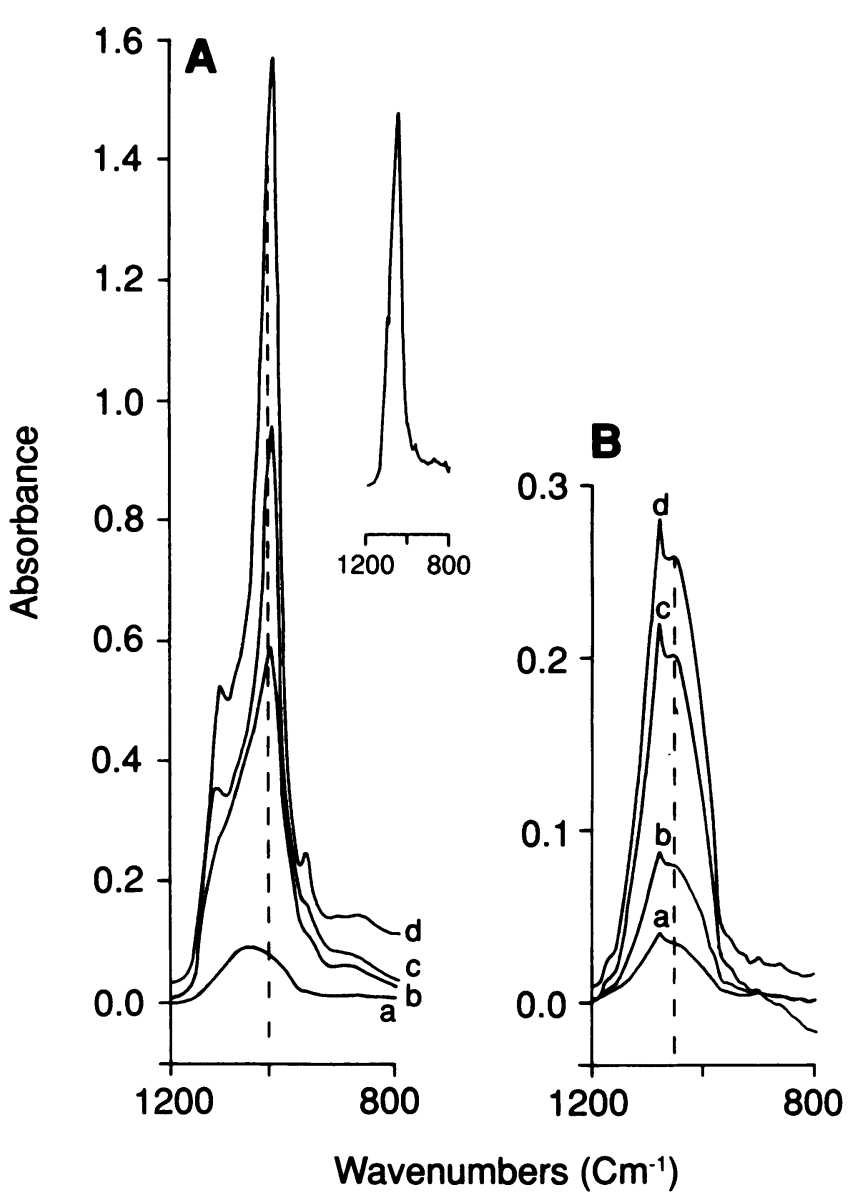

Figure 5. Effect of GCDC on the FTIR spectrum of maturing calcium phosphate. Calcium and phosphate were mixed and examined as described in Fig. 4. Spectra were obtained $(A)$ in the absence of GCDC at (a) $5 \mathrm{~min},(b) 85 \mathrm{~min},(c) 95 \mathrm{~min},(d) 240 \mathrm{~min}$; or $(B)$ in the presence of $2 \mathrm{mM} \mathrm{GCDC}$ at $(a) 1 \mathrm{~h},(b) 5 \mathrm{~h},(c) 48 \mathrm{~h},(d) 72 \mathrm{~h}$. The inset in $A$ shows the IR spectrum of standard HAP. The sharp unresolved peaks at $1,075 \mathrm{~cm}^{-1}$ in $B$ are caused by GCDC.

visible by $75 \mathrm{~min}$ (not shown). Otherwise the precipitation process did not markedly differ from the control situation, which was again consistent with earlier observations (Fig. 2).

Effect of GCDC on the stoichiometry of calcium phosphate precipitates. The FTIR spectrum of control precipitates after $I_{t}$ was very similar, but not identical, to the spectrum of the HAP standard, which suggested that nonapatite material may be contained in the precipitate. Precipitates were collected by filtration $(0.22 \mu \mathrm{m}) 30 \mathrm{~min}$ before $I_{\mathrm{t}}$ (phase 1 ) and by centrifugation $30 \mathrm{~min}$ after $I_{\mathrm{t}}$ (phase 3 ). Half of each precipitate was washed with $10 \mathrm{ml}$ of $10 \mathrm{mM}$ Tris buffer, $\mathrm{pH} 7.50$ and washed and unwashed precipitates were analyzed for calcium and phosphorus content (Table II). The unwashed phase 1 precipitates all had $\mathrm{Ca} / \mathrm{P}$ ratios close to 1 , which indicated that most of the phosphate was present as $\mathrm{HPO}_{4}^{2-}$. The $\mathrm{Ca} / \mathrm{P}$ ratio of unwashed phase 1 precipitates increased with increasing GCDC concentration. Washing the phase 1 precipitates caused the $\mathrm{Ca} / \mathrm{P}$ ratio to increase, suggesting that the interior of the particles was relatively less rich in $\mathrm{HPO}_{4}^{2-}$, although this must be a tentative interpretation because the small amount of material remaining after washing resulted in relatively large errors. The washed phase 3 samples all had $\mathrm{Ca} / \mathrm{P}$ ratios suggestive of HAP (1.67). 
Table II. Effect of GCDC on Ca/P Ratios (Mean $\pm S D$ ) of Washed and Unwashed Precipitates from Phases 1 and 3

\begin{tabular}{lccccc}
\hline & \multicolumn{2}{c}{ Phase 1 } & & \multicolumn{2}{c}{ Phase 3 } \\
\cline { 2 - 3 } \cline { 5 - 6 } GCDC & Unwashed & Washed & & Unwashed & Washed \\
\hline$m M$ & & & & & \\
0 & $1.00 \pm 0.07$ & $1.19 \pm 0.14^{*}$ & & $1.66 \pm 0.02$ & $1.73 \pm 0.03^{\ddagger}$ \\
0.5 & $1.00 \pm 0.06$ & $1.22 \pm 0.18^{*}$ & & $1.55 \pm 0.02$ & $1.69 \pm 0.04^{\xi}$ \\
1.0 & $1.05 \pm 0.08$ & 1.26 & & $1.67 \pm 0.05$ & $1.71 \pm 0.05^{*}$ \\
1.5 & $1.05 \pm 0.08$ & ND & & $1.71 \pm 0.03$ & $1.71 \pm 0.04^{*}$ \\
2.0 & $1.18 \pm 0.05$ & 1.50 & & - & - \\
\hline
\end{tabular}

$\mathrm{Ca}^{2+}$ and phosphate (4 $\mathrm{mM}$ final concentration of each) and increasing concentrations of GCDC were mixed together at $\mathrm{pH} 7.3, \mu=0.15$, $37^{\circ} \mathrm{C}$. Precipitates were collected both $30 \mathrm{~min}$ before (phase 1) and $30 \mathrm{~min}$ after (phase 3) $I_{t}$. Precipitates were analyzed either before or after washing with $10 \mathrm{mM}$ Tris, $\mathrm{pH}$ 7.5. In some cases, only one washed phase 1 sample was assayed because of losses during washing. Where SD values are given, at least three samples were assayed. Significance between washed and unwashed samples was assessed by $t$ test: ${ }^{*} \mathrm{NS} ;{ }^{\ddagger} P<0.05 ;{ }^{5} P<0.005$.

In the control phase 3 precipitates, washing caused the $\mathrm{Ca} / \mathrm{P}$ ratio to increase, which suggested that a surface layer, relatively rich in phosphate, had been removed from the precipitate. GCDC, $0.5 \mathrm{mM}$, decreased the $\mathrm{Ca} / \mathrm{P}$ ratio of unwashed phase 3 precipitates in comparison with the control, but 1 and $1.5 \mathrm{mM}$ GCDC progressively increased the $\mathrm{Ca} / \mathrm{P}$ ratio, until with 1.5 mM GCDC unwashed and washed precipitates did not differ. From these data we calculated the $\mathrm{Ca} / \mathrm{P}$ ratio of the surface fractions removed by washing from phase 3 precipitates, and plotted this ratio against induction time (Fig. 6). A low $\mathrm{Ca} / \mathrm{P}$ ratio (i.e., phosphate-rich surface fraction) was associated with a short $I_{t}$, and as the ratio approached the value for HAP (1.67), very small changes in composition were correlated with large changes in $I_{t}$.

\section{Discussion}

Except under very dilute conditions (17) the formation of HAP proceeds through a transitional ACP phase (18) by a process of dissolution and reprecipitation (6), rather than by solid-phase

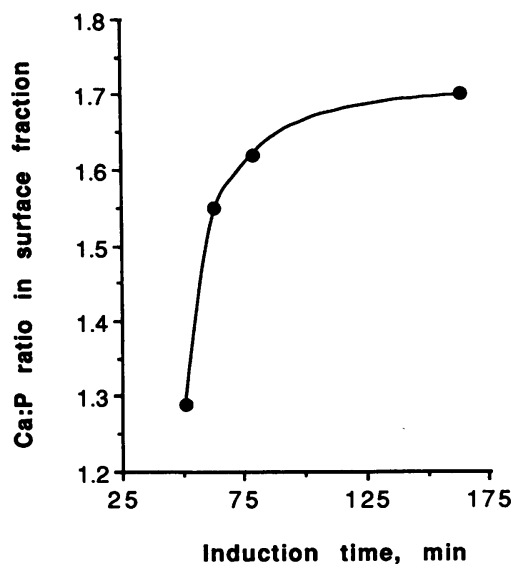

Figure 6. Correlation of induction time with the $\mathrm{Ca} / \mathrm{P}$ ratio of hydroxyapatite surface fraction. The composition of the surface fraction of hydroxyapatites formed in the presence of increasing concentrations of GCDC (0-1.5 mM) was calculated from the data in Table II and plotted against values of induction time obtained from turbidity measurements. transition (19), and the rate of conversion is proportional to the crystal content of the precipitate (20). The precise structural detail and chemical composition of both $\mathrm{ACP}$ and its transition products vary considerably $(18,21-23)$. Our results clearly show that low concentrations $(\sim 2 \mathrm{mM})$ of GCDC, but not TC, strongly inhibit the transformation of ACP to HAP. Although bile acids reduce $\mathrm{Ca}^{2+}$ activity $(24,25)$, and glycineconjugated bile acids are more effective than taurine conjugates (25), this is not the mechanism by which GCDC prevented HAP formation, since inhibition occurred at GCDC concentrations that did not measurably affect $\mathrm{Ca}^{2+}$ activity.

$A$ range of molecules and ions, including proteoglycans (26); phytates (27); $\mathrm{Mg}^{2+}, \mathrm{Sr}^{2+}$, and $\mathrm{Zn}^{2+}$ (28); ATP (29); pyrophosphate $(28,30)$; casein $(31)$; and phospholipids (32), inhibit HAP formation. Williams and Sallis (33) proposed that the minimum requirement for strong inhibition by anionic species is the possession of one phosphate group and one other acidic group, which may be either phosphate or carboxylic acid. Molecules with multiple carboxylic groups also inhibit, but the minimum number of groups required is unknown (34). Inhibition of HAP formation by GCDC appears reasonable because in the aggregated state GCDC should mimic a polycarboxylated molecule. The need for multiple carboxyl groups suggests that the action of submicellar concentrations of GCDC requires dimers or higher multimers. That GCDC caused potent inhibition at 2 $\mathrm{mM}$, a concentration at which very few micelles would be anticipated, also suggests submicellar multimers may be the active species. Experiments with dehydrocholic acid confirmed that partial inhibition could be caused by bile acid dimers, but 80 $\mathrm{mM}$ dehydrocholic acid was much less effective than $2 \mathrm{mM}$ GCDC, and, unlike GCDC, the effect of dehydrocholic acid was associated with reduced $\mathrm{Ca}^{2+}$ activity. The effect of dehydrocholic acid on $\mathrm{Ca}^{2+}$ activity was unexpected. Taurodehydrocholic acid does not affect $\mathrm{Ca}^{2+}$ activity as measured by the $\mathrm{Ca}^{2+}$-sensitive electrode (35), and therefore the present results may reflect our use of different methodology for measuring $\mathrm{Ca}^{2+}$ activity (11). It is unknown whether the greater inhibiting power of GCDC derives from its ability to form higher multimers, or from specific structural differences that may enhance the ability of GCDC dimers to interact with HAP. Dehydrocholic acid differs from GCDC in two important ways: the ring substituents are equatorial keto groups rather than axial hydroxyl groups, and the carboxyl-bearing side-chain is shorter. The presence of hydroxyl groups in GCDC may be important, since citric acid is an inhibitor of HAP formation, although its dehydroxyl and dehydro derivatives are inactive (36). The current data do not allow a definite conclusion to be drawn about the size of the GCDC aggregate required to inhibit HAP formation.

Williams and Sallis (12) proposed that most inhibitors could be divided into two classes: type I act principally by binding to HAP crystal embryos and poisoning them; type II function by decreasing the lability of ACP. Both types delay HAP formation, but only type I inhibitors reduce the amount of HAP formed (12). Because GCDC both delayed and reduced the production of HAP, this bile acid is a type I inhibitor. This conclusion is consistent with our observation that GCDC has a higher affinity for HAP than for ACP (Qiu, unpublished observations).

Both $\mathrm{ACP}$ and $\mathrm{HAP}$ contain $\mathrm{HPO}_{4}^{2-}$-rich surface fractions that can be removed by washing (18). The difference in $\mathrm{Ca} / \mathrm{P}$ ratios between unwashed and washed hydroxyapatites was a 
function of GCDC concentration, and the amount of surface phosphate in the precipitate was reduced by GCDC concentrations $>0.5 \mathrm{mM}$. The amount of surface phosphate could be reduced either by stabilization, so that it could not be removed by washing, or by inhibition of the initial accumulation of acid phosphate on the particle surface. The general upward trend in the $\mathrm{Ca} / \mathrm{P}$ ratio of unwashed precipitates with increasing GCDC concentration supports the second interpretation. We have shown that GCDC binding to $\mathrm{HAP}$ is inhibited by $\mathrm{HPO}_{4}^{2-}$, but not by $\mathrm{H}_{2} \mathrm{PO}_{4}^{-}$, suggesting that $\mathrm{GCDC}$ and $\mathrm{HPO}_{4}^{2-}$ compete for common binding sites (37). Such competition for binding sites on newly formed HAP embryos should poison the crystal surface. This competition is reflected in our observation that a phosphate-rich surface fraction is correlated with a short $I_{t}$. At low GCDC concentrations, the competition may be enough only to slow the rate of crystal growth, but at higher concentrations, because of an increase in either the size or number of aggregates, the degree of poisoning may be so great that growth is prevented and the HAP embryos redissolve before they constitute a sufficient fraction of the total calcium phosphate precipitate to be detected by FTIR. To the observer this process would appear as stabilization of the amorphous state.

It is unknown why GCDC shows greater binding than TC to ACP (7) and HAP (38). However, because all the glycineconjugated bile acids investigated, as well as unconjugated deoxycholic acid, bound to a much greater extent than any taurine conjugate, a carboxylic acid group may favor binding (7). In addition, glycocholic acid bound to ACP (7) and HAP (38) less well than did the glycine-conjugated dihydroxy bile acids, thus the ineffectiveness of TC may be related to both its conjugation and its hydroxylation pattern. The increased affinity of $\mathrm{Ca}^{2+}$ for glycine conjugates over taurine conjugates has been attributed to more extensive hydration of the latter (25). However, in the present situation bile acid binding is not occurring to isolated $\mathrm{Ca}^{2+}$ ions in solution, but rather to the HAP surface, which consists of regions of alternating positive and negative polarity. Therefore, if adjacent anionic groups of the bile acid aggregate are not arrayed in a manner that brings them into apposition with regions of positive polarity, they will likely interact with negatively charged areas and be repelled. Under this scenario, GCDC aggregates possess anionic groups that mirror the spacing of calcium ions on the HAP surface, whereas the sulphonate groups of TC aggregates are misaligned. In experiments with mixed TC/GCDC solutions, TC was incorporated into GCDC aggregates and attenuated the inhibition of HAP formation. This effect is probably caused by increasing amounts of TC inducing progressively severe misalignment of anionic groups. The observation that the action of a given submicellar concentration of GCDC can be augmented by small amounts of TC sufficient to cause increased bile acid aggregation indicates that the deleterious effects of small amounts of TC are insufficient to offset the advantage of increased aggregation.

The ability of mixed TC/lecithin micelles to inhibit calcium phosphate formation (5) is probably caused by binding to ACP or HAP nuclei initiated by the lipid phosphate groups. TC and other taurine-conjugated bile acids, as well as GCDC, inhibit the precipitation of calcium bilirubinate $(4,39)$. The fact that some bile acids inhibit precipitation of only some calcium salts (TC can inhibit calcium bilirubinate, but not HAP) again suggests that inhibition of calcium salt precipitation does not necessarily occur as a result of direct action on the common cation, calcium, in solution. In some instances inhibition may be mediated by specific interactions between the bile acid and the anion, either in solution or in a microprecipitate. In other cases, the target may be calcium ions incorporated into a crystal embryo. The spacing of these calcium ions is a function of crystal type, so small differences in the structures of aggregates of different bile salts may mean that the anionic groups in any given bile salt aggregate are optimally aligned to interact preferentially with the calcium ions of a particular crystal. If this is true, changes in the bile acid composition of bile over time may affect the relative proportions of the various calcium salts that will be laid down in developing gallstones. This hypothesis could partially explain the differences in composition observed in moving from the center to the surface in many gallstones (2).

Finally, we emphasize that the effect of bile acids on the precipitation of calcium salts cannot be considered in isolation, but must eventually be integrated into a more comprehensive model in which the role of other components of bile, such as phospholipids (32) and glycoproteins (3), and the presence of distinct microenvironments within the gallbladder (2) are taken into account.

\section{Acknowledgments}

We wish to thank Alice Cullu for editorial assistance, and Dr. Nancy Alcock for performing the total calcium determinations.

This study was supported in part by grant AM-16549 from the National Institutes of Health and grants from the Sealy and Smith Foundation.

\section{References}

1. Womack, N. A., R. Zeppa, and G. L. Irvin. 1963. The anatomy of gallstones. Ann. Surg. 157:670-686.

2. Crowther, R. S., and R. D. Soloway. 1990. Pigment gallstone pathogenesis: from man to molecules. Semin. Liver Dis. 10:171-180.

3. Shimizu, S., B. Sabsay, A. Veis, J. D. Ostrow, R. V. Rege, and L. G. Dawes. 1989. Isolation of an acidic protein from cholesterol gallstones, which inhibits the precipitation of calcium carbonate in vitro. J. Clin. Invest. 84:1990-1996.

4. Angelico, M., S. C. De Sanctis, C. Gandin, and D. Alvaro. 1990. Spontaneous formation of pigmentary precipitates in bile salt-depleted rat bile and its prevention by micelle-forming bile salts. Gastroenterology. 98:444-453.

5. Sutor, D. J., and J. M. Percival. 1976. Presence or absence of inhibitors of crystal growth in bile. 1 . Effect of bile on the formation of calcium phosphate, a constituent of gallstones. Gut. 17:506-510.

6. Eanes, E. D., J. D. Termine, and M. U. Nylen. 1973. An electron microscopic study of the formation of amorphous calcium phosphate and its transformation to crystalline apatite. Calcif. Tissue Res. 12:143-158.

7. Van Der Meer, R., and H. T. De Vries. 1985. Differential binding of glycine- and taurine-conjugated bile acids to insoluble calcium phosphate. Biochem. J. 229:265-268.

8. Crowther, R. S., S.-M. Qiu, G. Wen, N. Alcock, N.-K. Hong, and R. D. Soloway. 1990. A turbidimetric study of the precipitation of calcium phosphate. Gastroenterology. 98:A245. (Abstr.)

9. Crouch, S. R., and H. V. Malmstadt. 1967. A mechanistic investigation of molybdenum blue method for determination of phosphate. Anal. Chem. 39:1084-1089.

10. Chattopadhyay, A., and E. London. 1984. Fluorimetric determination of critical micelle concentration avoiding interference from detergent charge. Anal. Biochem. 139:408-412.

11. Lichtenberg, D., E. Werker, A. Bor, S. Almog, and S. Nir. 1988. Precipitation of calcium palmitate from bile salt-containing dispersions. Chem. Phys. Lipids. 48:231-243.

12. Williams, G., and J. D. Sallis. 1982. Structural factors influencing the ability of compounds to inhibit hydroxyapatite formation. Calcif. Tissue Int. 34:169-177.

13. Roda, A., A. F. Hofmann, and K. J. Mysels. 1983. The influence of bile salt structure on self-association in aqueous solutions. J. Biol. Chem. 258:63626370 . 
14. Small, D. M. 1971. The physical chemistry of cholanic acids. In The Bile Acids, Volume I. P. P. Nair and D. Kritchevsky, editors. Plenum Press, New York. 249-356.

15. Termine, J. D., and D. R. Lundy. 1974. Vibrational spectra of some phosphate salts amorphous to X-ray diffraction. Calcif. Tissue Res. 15:55-70.

16. Nancollas, G. H. 1984. Crystallization in bile. Hepatology (Baltimore). $4: 169 \mathrm{~S}-172 \mathrm{~S}$.

17. Boskey, A. L., and A. S. Posner. 1976. Formation of hydroxyapatite at low supersaturation. J. Phys. Chem. 80:40-45.

18. Termine, J. D., and E. D. Eanes. 1972. Comparative chemistry of amorphous and apatitic calcium phosphate preparations. Calcif. Tissue Res. 10:171197.

19. West, V. C. 1971. Observations on phase transformation of a precipitated calcium phosphate. Calcif. Tissue Res. 7:212-219.

20. Eanes, E. D., and A. S. Posner. 1965. Kinetics and mechanism of conversion of noncrystalline calcium phosphate to crystalline hydroxyapatite. Trans N.Y. Acad. Sci. 28:233-241.

21. Cheng, P.-T., and K. P. H. Pritzker. 1983. Solution $\mathrm{Ca} / \mathrm{P}$ ratio affects calcium phosphate crystal phases. Calcif. Tissue Int. 35:596-601.

22. Eanes, E. D., and J. L. Meyer. 1977. The maturation of crystalline calcium phosphates in aqueous suspensions at physiologic pH. Calcif. Tissue Res. 23:259-269.

23. Tung, M. S., and W. E. Brown. 1983. An intermediate state in hydrolysis of amorphous calcium phosphate. Calcif. Tissue Int. 35:783-790.

24. Moore, E. W., L. Celic, and J. D. Ostrow. 1982. Interactions between ionized calcium and sodium taurocholate: bile salts are important buffers for prevention of calcium-containing gallstones. Gastroenterology. 83:1079-1089.

25. Rajagopalan, N., and S. Lindenbaum. 1984. Counter-ion binding by bile acid solutions. Hepatology (Baltimore). 4:110S-114S.

26. Blumenthal, N. C., A. S. Posner, L. D. Silverman, and L. C. Rosenberg 1979. Effect of proteoglycans on in vitro hydroxyapatite formation. Calcif. Tissue Int. 27:75-82.

27. Van Den Berg, C. J., L. F. Hill, and S. W. Stanbury. 1972. Inositol phosphates and phytic acid as inhibitors of biological calcification in the rat. Clin. Sci. (Oxf.). 43:377-383.

28. Root, M. J. 1990. Inhibition of the amorphous calcium phosphate phase transformation reaction by polyphosphates and metal ions. Calcif. Tissue Int. 47:112-116.

29. Termine, J. D., and K. M. Conn. 1976. Inhibition of apatite formation by phosphorylated metabolites and macromolecules. Calcif. Tissue Res. 22:149157.

30. Fleisch, H., and S. Bisaz. 1962. Isolation from urine of pyrophosphate, a calcification inhibitor. Am. J. Physiol. 203:671-675.

31. Termine, J. D., R. A. Peckauskas, and A. S. Posner. 1970. Calcium phosphate formation in vitro II. Effects of environment on amorphous-crystalline transformation. Arch. Biochem. Biophys. 140:318-325.

32. Wuthier, R. E., and E. D. Eanes. 1975. Effect of phospholipids on the transformation of amorphous calcium phosphate to hydroxyapatite in vitro. $\mathrm{Cal}$ cif. Tissue Res. 19:197-210.

33. Williams, G., and J. D. Sallis. 1979. Structure-activity relationship of inhibitors of hydroxyapatite formation. Biochem. J. 184:181-184.

34. Hay, D. I., E. C. Moreno, and D. H. Schlesinger. 1979. Phosphoproteininhibitors of calcium phosphate precipitation from salivary secretions. Inorg. Perspect. Biol. Med. 2:271-285.

35. Moore, E. W., A. F. Hofmann, C. D. Schteingart, and H.-T. Ton-Nu. 1990. High-affinity premicellar $\mathrm{Ca}^{++}$-binding to bile salts: II. Structure-activity relationships. Hepatology (Baltimore). 12:998. (Abstr.)

36. Tew, W. P., C. Mahle, J. Benavides, J. E. Howard, and A. L. Lehninger. 1980. Synthesis and characterization of phosphocitric acid, a potent inhibitor of hydroxyapatite crystal growth. Biochemistry. 19:1983-1988.

37. Crowther, R. S., N.-K. Hong, S.-M. Qiu, and R. D. Soloway. 1991. Glycochenodeoxycholic acid inhibits calcium hydroxyapatite formation by competetively inhibiting $\mathrm{HPO}_{4}^{2-}$ binding to the particle surface. Gastroenterology. 100:A733. (Abstr.)

38. Qiu, S.-M., R. S. Crowther, and R. D. Soloway. 1991. The ability of bile acids to inhibit calcium hydroxyapatite formation is reflected in their apatite binding affinity. Gastroenterology. 100:A787. (Abstr.)

39. Hong N.-K, R. D. Soloway, R. S. Crowther, F Liu, S.-M. Qiu, H. Guo, N. Alcock, and J.-G. Wu. 1990. Chenodeoxycholate conjugates are more effective than taurocholate in inhibiting the precipitation of calcium bilirubinate. Gastroenterology. 98:A250. (Abstr.) 